
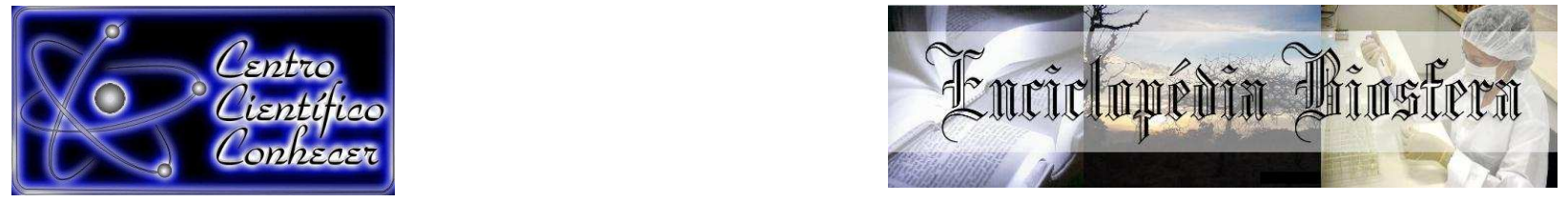

\title{
SISTEMA COLETOR DE DADOS PORTÁTIL PARA A AQUISIÇÃO DO ÍNDICE DE TEMPERATURA E UMIDADE EM ALOJAMENTOS DE FRANGOS COBB
}

Mario Mollo Neto ${ }^{1}$, Willian Aparecido Leoti Zanetti ${ }^{2}$, Larissa Talita de Oliveira ${ }^{2}$.

1- Prof. Dr. do Curso de Engenharia de Biossistemas, UNESP, Tupã/SP - Brasil mariomollo@tupa.unesp.br

2- Discente do Curso de Engenharia de Biossistemas UNESP, Tupã/SP - Brasil

Recebido em: 08/04/2016 - Aprovado em: 30/05/2016 - Publicado em: 20/06/2016 DOI: 10.18677/Enciclopedia_Biosfera_2016_151

\begin{abstract}
Um sistema coletor de dados inteligente microcontrolado, foi desenvolvido e avaliado para a monitoração de indicadores de conforto ambiental dos galpões de produção de aves Cobb. O objetivo deste artigo tem seu foco em produzir um dispositivo portátil que, automaticamente e de maneira autônoma, possa calcular o indicador referente ao ITU que é o índice que correlaciona a temperatura e a umidade do ar presente no ambiente do alojamento, armazenando os dados digitalmente em mídia removível, para posterior avaliação dos indicadores, gerando informações de classificação de patamares relativos ao conforto das aves e que orientem ações para evitar o estresse gerado pelo calor. Para tal foram utilizados uma placa eletrônica microcontrolada, um sensor de temperatura eletrônico montado em um globo negro construído em cobre, um módulo sensor de temperatura e de umidade relativa do ar, um módulo sensor de pressão atmosférica, um módulo de relógio em tempo real, um módulo para leitura e escrita em cartões SD. O firmware foi construído com base em um software dedicado para a placa adotada e um modelo reduzido em escala de uma instalação avícola. Os resultados que foram alcançados comprovam que o uso de sistemas microcontrolados aliados ao uso de sensores eletrônicos, podem gerar eficientes ferramentas de suporte a decisão para os gestores de processos.
\end{abstract}

RESUMO

PALAVRAS- CHAVE: estresse de calor, frango Cobb, sensores.

\section{PORTABLE DATA COLLECTION SYSTEM FOR THE ACQUISITION OF TEMPERATURE AND HUMIDITY INDEX IN THE BROILER SHEDS}

\begin{abstract}
A intelligent microcontroller data collector system, was developed and evaluated for monitoring environmental comfort indicators of Cobb poultry production sheds. The purpose of this article is focused on producing a portable device that automatically and autonomously, can calculate the indicator for the ITU which is the index that correlates the temperature and humidity present in the housing environment by storing data digitally on removable media, for further evaluation of the indicators, generating levels of classification for information regarding the comfort of the birds
\end{abstract}


and to guide actions to prevent stress generated by heat. For this been used one microcontrolled electronic board, an electronic temperature sensor mounted on a black globe built in copper, an air temperature and relative humidity sensor module, an atmospheric pressure sensor module, a clock in real time module, a module for reading and writing on SD cards. The firmware was built on a software dedicated to the adopted board and a small scale model of a poultry facility. The results that have been achieved show that the use of microcontroller coupled with the use of electronic sensor systems can generate efficient decision support tools for process managers.

KEYWORDS: heat stress, poultry, sensors.

\section{INTRODUÇÃO}

A ABPA (2014), destaca que a cadeia produtiva do frango Cobb, no Brasil em 2014, movimentou o valor de US\$ 8,5 Bilhões de dólares. Considerando que isto equivale a $1,5 \%$ do o Produto Interno Bruto (PIB) de 2014 e, segundo o IBGE (2012), a cadeia representou 3,8\% do total do PIB Agropecuário, fatos que destacam a importância desta cadeia produtiva para o Brasil.

Conforme o documento publicado pela Confederação da Agricultura e ecuária do Brasil (CNA, 2013) a carne de frango, em 2013, continuou sendo a preferida dos brasileiros, registrando consumo de 46 quilos per capita ao ano. A principal razão desse bom desempenho, segundo a CNA, foi o aumento do poder aquisitivo das classes $\mathrm{C}$, $\mathrm{D}$ e $\mathrm{E}$, ocorrida no passado recente.

Sob este aspecto PEREIRA et al. (2011) dizem que o ambiente de produção para frangos Cobb é diferenciado em físico, aéreo, térmico, biológico, acústico e social, e destaque também é dado por NASCIMENTO et al. (2011) que afirmam que a exposição ao estresse térmico tem um impacto negativo na produtividade, afetando o consumo de ração, ganho de peso e conversão alimentar e, apontam que neste cenário, a produção de aves hoje em dia requer um certo grau de tecnologia que envolve a utilização intensa de material resultante da eficiência econômica.

Pode-se destacar, ainda, que no sistema de criação, os fatores térmicos influenciam diretamente os frangos Cobb, em virtude da alteração na manutenção da homeotermia (BARBOSA FILHO et al., 2009).

Um dos principais fatores que impactam a eficiência da produção em locais quentes é o estresse devido à temperatura ambiente (ABDELQADER \& ALFATAFTAH, 2014). Uma das consequências do estresse é a mudança do equilíbrio ácido-base com o aparecimento da alcalose respiratória à medida que a interação entre a umidade relativa e temperatura ambiente ultrapassam a faixa da termoneutralidade (BORGES et al., 2003).

A determinação do índice proposto por THOM (1959) que é o Índice de Temperatura e Umidade (ITU) será realizada com base na descrição dada por MOLLO NETO et al. (2015), utilizando-se, também, da metodologia proposta por WILHELM (1976). NIENABER \& HAHN (2007), afirmam que os valores de ITU, seriam válidos para animais domésticos de forma global, aplicando-se assim aos frangos Cobb, objeto desta pesquisa.

NIENABER \& HAHN (2007) construíram uma tabela para a indicação dos índices de ITU para os animais consoante com o impacto que representa para os animais de forma global. Os autores apresentam as faixas que definem as classes deste estudo como sendo "Normal" para valores de ITU menores que 74; "Alerta" para valores de ITU entre 74 e 79; "Perigo" para valores de ITU entre 79 e 84 e "Emergência" para valores de ITU maiores que 84. 
Observa-se que neste mercado, acontece o uso crescente de automação, agora comuns para o processamento de aves, resultando em produto final de alta qualidade (BARBUT, 2014). O mesmo autor destaca que este é um desafio constante, porque os equipamentos requerem novos tipos de sensores e sistemas de controle únicos que substituem operações manuais tradicionais e ajudando nos equipamentos em operação automática.

A agricultura de precisão é o uso da ciência e tecnologia para aprimorar a produção (MESAS-CARRASCOSA et al., 2015). Os mesmos autores reforçam que o uso destas tecnologias com a inserção de sensores aprimoram a agricultura dandoIhe maior flexibilidade e economia devido à precisão obtida.

Destacam, também, que os recentes avanços nas comunicações e eletrônica permitiram o desenvolvimento e produção de custo reduzido e baixo consumo de energia, de sensores multifuncionais, que são pequenos em tamanho e podem se comunicar de maneira inteligente e barata, podendo ser instalados em grandes quantidades, proporcionando enormes oportunidades a gestão de ambientes.

Levando este contexto em conta, a melhor seleção do hardware que compõe os nós sensores é uma consideração importante para qualquer implantação de sistemas de agrícolas que trabalham com precisão.

A análise de diferentes arquiteturas de hardware podem ser realizadas observando-se os parâmetros gerais, referentes ao processador e a memória, sua capacidade de comunicação, o apoio a ligação dos sensores e o correspondente consumo de energia. Uma das principais características na seleção dos nós (hardware de microcontrolador/sensor) são seus parâmetros físicos, peso, tamanho e preço (MRIDULA et al., 2013).

O Arduino, com chip AVR da ATMEL, foi escolhido para esta pesquisa devido a sua facilidade de utilização e disponibilidade de recursos na web.

Outros trabalhos foram já desenvolvidos com o uso deste mesmo hardware, por KAMOGAWA \& MIRANDA (2013); CARVALHO et al. (2014); CARVALHO \& AMORIM (2014); CANDELAS et al. (2015); HARIKRISHNAN (2015); SÁNCHES et al. (2015); ALI et al. (2016); FATEHNIA et al. (2016); VARANIS et al. (2016).

Assim, este artigo teve como objetivo em produzir um dispositivo portátil que, automaticamente e de maneira autônoma, possa coletar o índice de temperatura e umidade (ITU) do ambiente do alojamento, armazenando os dados digitalmente em mídia removível, para posterior avaliação dos indicadores, gerando informações de classificação de patamares relativos ao conforto das aves e que orientem ações para evitar o estresse gerado pelo calor.

\section{MATERIAL E MÉTODOS}

Iniciaram-se os trabalhos com a busca e organização de literatura correlacionada ao tema disponível nas bases mais conhecidas de pesquisa.

A seguir, foram identificados os sensores para o protótipo.

O hardware foi construído, soldando-se os componentes e módulos eletrônicos em uma placa tipo shield, que é montada por meio de conectores por sobre a placa base do Arduino Mega, e foi testado de forma a confirmar todo o seu funcionamento, inclusive a montagem do leitor de cartão SD.

Aplicando-se a ferramenta disponibilizada por ARDUINO-SOFTWARE (2014), foi codificado o firmware para a leitura sequenciada dos sensores, do leitor de cartão $\mathrm{SD}$ e do relógio de tempo real (RTC) para registro das datas e horas em que cada leitura foi realizada. 
Com base nos valores das variáveis medidas pelos sensores, o microcontrolador calcula e armazena com os respectivos valores de data e hora para cada registro, em mídia removível, os valores do índice de temperatura e umidade (ITU), e registra uma classificação indicando os patamares e se os mesmos encontram-se dentro dos limites de conforto térmico propostos por NIENABER \& HAHN (2007).

O experimento para a validação do hardware e firmware (software embarcado no chip microcontrolador) projetado, foi realizado no câmpus da UNESP da cidade de Tupã, conforme as recomendações encontradas em NÄÄS et al. (2001).

O protótipo, após a confirmação de seu correto funcionamento, foi instalado para a aquisição dos valores das variáveis climáticas para a sua validação no interior do modelo de instalação avícola.

Utilizou-se um modelo reduzido em escala distorcida que reproduz as instalações avícolas, construídos na direção Leste-Oeste, situado à latitude $21^{\circ} 55^{\prime}$ 39" S, 50²9' 30" W, altitude 495 m, classificação climática Cwa, sendo seu entorno constituído de área plana coberta de grama. As escalas utilizadas são 1:10 nas dimensões horizontais e 1:2 nas dimensões verticais. A Tabela 1 apresenta as dimensões do modelo utilizado.

TABELA 1. Dimensões adotadas para o modelo reduzido em escala distorcida que reproduz as instalações avícolas, utilizado no experimento.

\begin{tabular}{c|ccc}
\hline Escala & $1: 1$ (natural) & $1: 10$ (horizontal) & $1: 2$ (vertical) \\
\hline Largura & $14,00 \mathrm{~m}$ & $1,40 \mathrm{~m}$ & - \\
\hline Comprimento & $30,00 \mathrm{~m}$ & $3,00 \mathrm{~m}$ & - \\
\hline Pé direito & $3,00 \mathrm{~m}$ & - & $1,50 \mathrm{~m}$ \\
\hline Altura mureta & $0,20 \mathrm{~m}$ & - & $0,10 \mathrm{~m}$ \\
\hline
\end{tabular}

Fonte: Os Autores.

O equipamento construído foi instalado no modelo reduzido, sendo internamente posicionados os sensores para as coletas das variáveis climáticas desejadas.

O firmware do sistema, escrito em $C$ na interface disponibilizada por ARDUINO-SOFTWARE (2014), foi ajustado para a coleta num intervalo de 5 em 5 minutos e o algoritmo baseou-se nas variáveis climáticas coletadas e nos valores calculados de ITU enviando pela sua porta serial os dados para um computador PC e simultaneamente para o registro dos dados em um cartão de memória tipo $S D$, num arquivo do tipo .txt com separação por vírgulas, para futuro transporte e exportação para o Microsoft Excel para maiores analises.

O indicador do conforto ambiental (ITU) foi calculado no sistema embarcado, automaticamente logo após a cada uma das coletas e o algoritmo, com base nas regras proposta por WILHELM (1976), e as definições de registro das faixas de status do conforto térmico, segundo a tabela desenvolvida nos estudos de NIENABER \& HAHN (2007).

\section{RESULTADOS E DISCUSSÃO}

Com a pesquisa aos fabricantes de sensores eletrônicos, foi possível determinar quais seriam os sensores mais indicados para o projeto, então, foram escolhidos: o sensor de pressão BMP180, o sensor de umidade DHT11 e o sensor de temperatura LM35 inserido em um globo metálico de cobre. 
De posse dos sensores e suas especificações, adotou-se o módulo didático Arduino Mega 2560 como sendo a placa microprocessada para a pesquisa. Foi necessário montar uma placa adicional (Shield) para a conexão dos sensores escolhidos. Nesta mesma placa foram montados dois módulos, um para geração da base de tempo para registro em tempo real das datas e horas das medições e registros a serem efetuados e um módulo de gravação/leitura de cartões de mídia removível do tipo SD.

$\mathrm{Na}$ Figura 1 podem ser visualizadas as placas do protótipo construído e suas ligações na placa Shield já montada sobre a placa microprocessada Arduino Mega 2560.

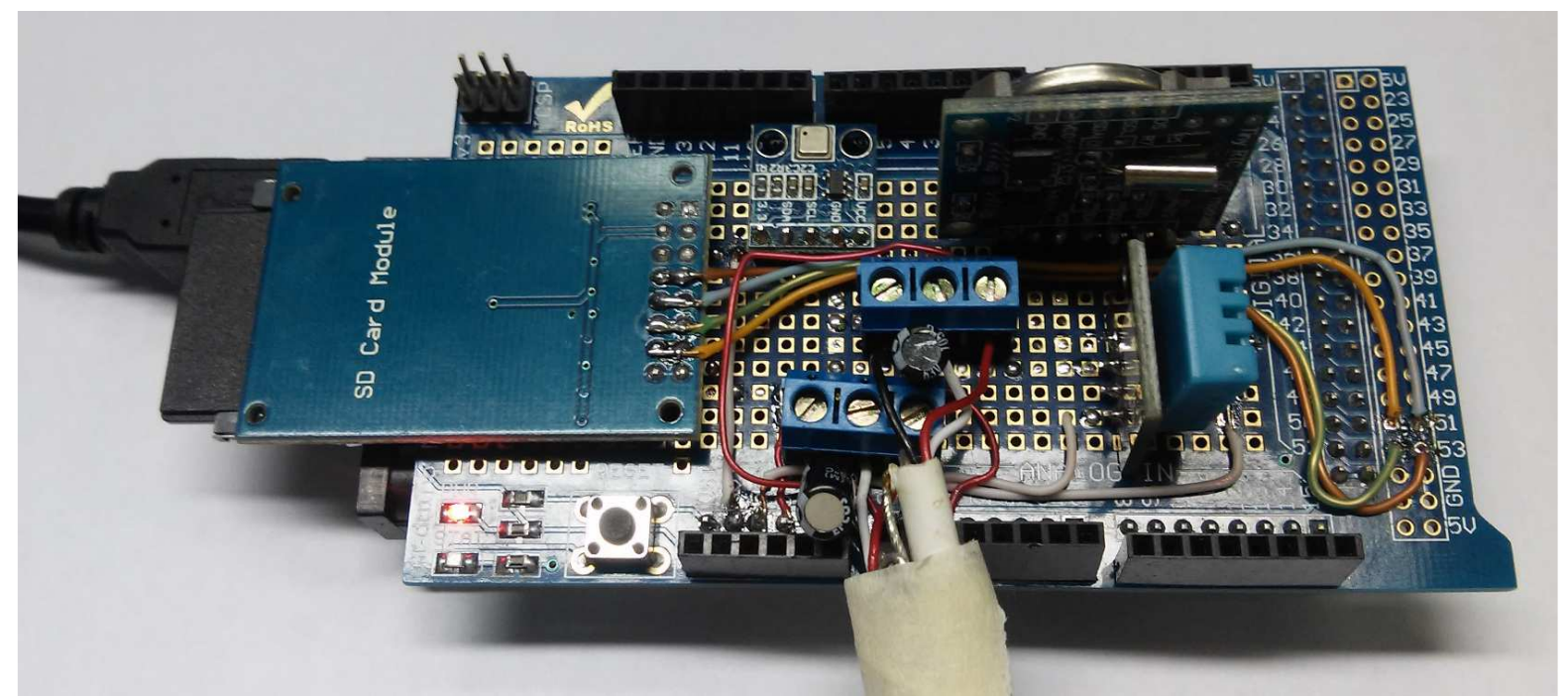

FIGURA 1. Placas eletrônicas do sistema de supervisão, coleta e armazenamento de dados projetado e construído para utilização em alojamentos de frangos. Fonte: Os autores.

Os módulos eletrônicos foram acoplados às entradas analógicas e à entrada serial do barramento $\mathrm{I} 2 \mathrm{C}$ da placa.

O firmware dedicado para o hardware desenvolvido, foi construído com a ferramenta fornecida por ARDUINO-SOFTWARE (2014), e o mesmo foi configurado para, inicilamente realizar um teste correspondente à existência ou não de um cartão SD inserido no módulo de leitura/escrita e verificar a sua integridade, informando se o mesmo está pronto, ou não para a coleta de dados.

Após o teste, a programação foi realizada de forma a apresentar em sua interface de canal de comunicação serial ao microcomputador PC a coleta de dados, paralelamente ao registro em cartão $\mathrm{SD}$, dos valores correspondentes das variáveis climáticas medidas e daquelas que foram calculadas.

As variáveis climáticas lidas pelos sensores foram:
a) Temperatura de Globo negro $\left({ }^{\circ} \mathrm{C}\right)$;
b) Umidade Relativa do ar (\%);
c) Temperatura do $\operatorname{ar}\left({ }^{\circ} \mathrm{C}\right)$;
d) Altitude (m);
e) Pressão Atmosférica $(\mathrm{kPa})$. 
As variáveis calculadas foram as seguintes:

a) Temperatura de Ponto de Orvalho $\left({ }^{\circ} \mathrm{C}\right)$;

b) Indicador ITU;

c) Status do indicador ITU nas classes definidas por NIENABER \& HAHN (2007).

No firmware foi, ainda, prevista a exibição na interface de comunicação serial, paralelamente com a gravação em cartão SD, das variáveis correspondentes a data e hora das coletas, assim como o dia da semana.

O circuito eletrônico representativo da composição do projeto realizado para a construção do equipamento proposto para a presente pesquisa, com os seus módulos integrados, é apresentado na Figura 2.
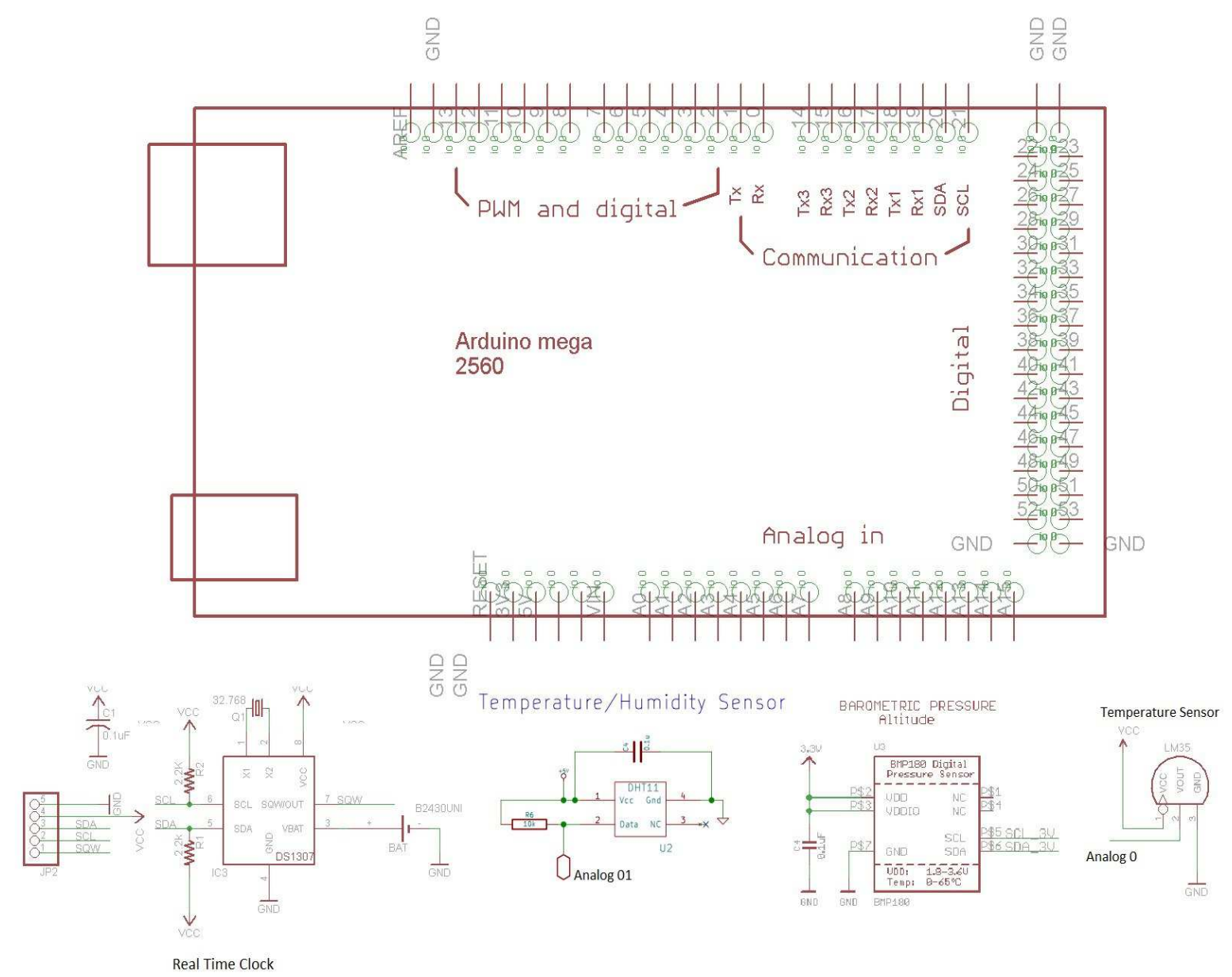

FIGURA 2. Circuito base e pinagens de conexões do módulo Mega 2560 projetado com os circuitos para cada um dos sensores - LM35, DHT11, BMP180 e o relógio de tempo real (RTC). Fonte: Os autores.

O sistema foi testado e verificado em bancada, e na sequência foi instalado no interior do modelo reduzido em escala distorcida que reproduz as instalações avícolas, e por meio de uma fonte de alimentação conversora de 110/220 Vca $12 \mathrm{Vcc}$, foi energizado, iniciando as coletas de dados e os devidos registros das variáveis climáticas. 
O sistema foi mantido no modelo reduzido em escala distorcida que reproduz as instalações avícolas por um período de uma semana coletando dados climáticos e calculando as variáveis para monitoramento de ITU e do seu correspondente status.

Ao ser removido do modelo reduzido em escala distorcida, o sistema retornou ao laboratório e o cartão SD foi removido para análise e observou-se que a coleta estava integra e todos os registros datados corretamente, sem interrupções, o que validou o seu satisfatório funcionamento.

Os resultados de medição obtidos podem ser apreciados na Figura 3, que apresenta a interface de visualização dos dados coletados em tempo real, obtidos da placa com os sensores e transmitidos pelo canal de comunicação serial.

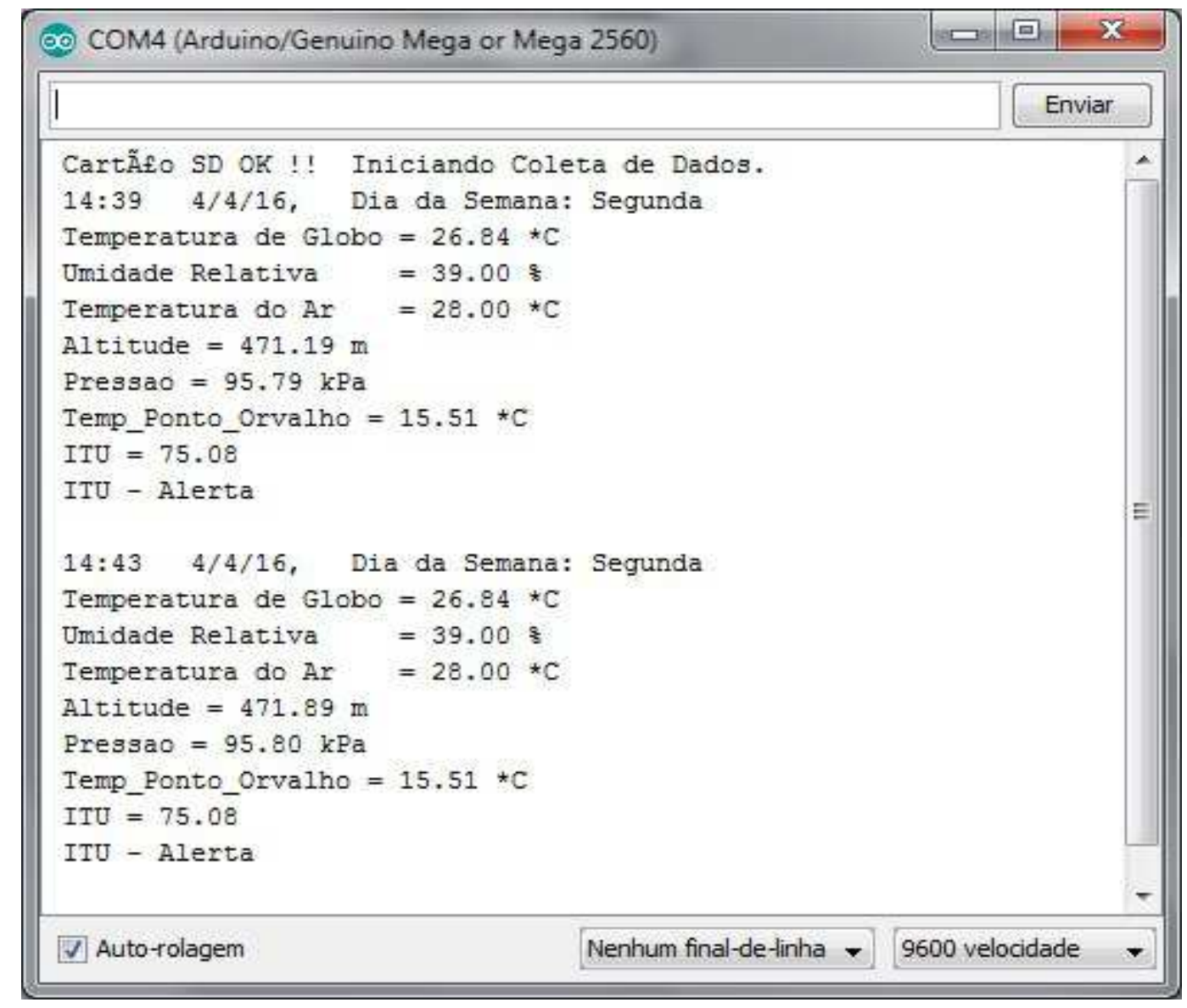

FIGURA 3. Listagem das leituras apresentada na tela do PC com resultados correspondentes às variáveis climáticas que foram gravados em arquivo .txt em cartão SD para futura exportação para análise. Fonte: Os autores.

Observou-se que o sistema é robusto e pode ser utilizado no interior de galpões de produção de aves Cobb e até mesmo de outras espécies, o que permite inferir que o sistema proposto permite gerar visualizaçõe e registros de dados do ambiente de produção como uma eficiente ferramenta de suporte a decisão para os gestores de processos mediante as classificações de conforto apresentadas.

O sistema conta, ainda, com uma baixa complexidade e com grandes possibilidades de redução de custos para monitoramento em tempo real, gerando ENCICLOPÉDIA BIOSFERA, Centro Científico Conhecer - Goiânia, v.13 n.23; p.1690 2016 
registros confiáveis que permitem ao usuário ter acesso em qualquer computador pessoal para análises posteriores.

\section{CONCLUSÕES}

Com a pesquisa bibliográfica realizada, aliada com os procedimentos metodológicos aplicados para projetar o coletor de dados, aplicando um microcontrolador AVR da ATMEL na versão MEGA 2560, com um shield de prototipagem, foi possível a montagem de um conjunto de componentes para sensoreamento, ajustados para a medição das variáveis meteorológicas necessárias ao cálculo do indicador de conforto pretendido.

O dispositivo, em seus testes de funcionamento, permaneceu coletando e armazenando digitalmente, em mídia removível, com boa precisão, os dados do ambiente do alojamento, e inferiu as condições do alojamento calculando o índice de temperatura e umidade (ITU) para as aves Cobb.

Com estes dados, passou a apresentar o status da variável calculada e indicando a sua classificação, permitindo, assim, aos gestores e criadores, um melhor suporte a decisão para determinar ações para mitigar o estresse gerado pelo calor em suas instalações para a produção de aves da genética Cobb.

Conclui-se também que o dispositivo gerado pode ser aplicado também para criatórios de aves de outras genéticas.

\section{AGRADECIMENTOS}

Todos os participantes desta pesquisa, agradecem ao CNPq pelo auxílio à pesquisa e a UNESP campus de Tupã pelo acesso às instalações e equipamentos.

\section{REFERÊNCIAS}

ABDELQADER, A., AL-FATAFTAH, A. R. Thermal acclimation of broiler birds by intermittent heat exposure. Journal of Thermal Biology, v. 39, n.1, p. 1-5, 2014. Disponível em: <http://www.sciencedirect.com/science/article/pii/S0306456513001204>. Acesso em: 24/05/2016. doi:10.1016/j.jtherbio.2013.11.001.

ABPA. Associação Brasileira de Proteína Animal, Avicultura brasileira panorama da avicultura nacional e perspectivas do Setor. Disponivel em: < http://www.agricultura.gov.br/arq_editor/file/PNSA/Reuni\%C3\%A30\%20PNSA_\%20_ Sanidade\%20Av\%C3\%ADcola-Fortaleza\%20Nacional_/2\%20Dr_\%20Ariel\%20\%20Panorama\%20da\%20avicultura\%20nacional\%20e\%20perspectivas\%20para\%2 0o\%20setor.pdf> Acesso em: 18/05/2016.

ALI, A. S.; ZANZINGER, Z.; DEBOSE, D.; STEPHENS, B. Open Source Building Science Sensors (OSBSS): A low-cost Arduino-based platform for long-term indoor environmental data collection. Building and Environment, v. 100, n.1, p. 114-126, 2016.

em: <http://www.sciencedirect.com/science/article/pii/S0360132316300476> Acesso em: 24/05/2016. doi:10.1016/j.buildenv.2016.02.010

ARDUINO-SOFTWARE. Download the Arduino Software. Disponível em: < http://arduino.cc/en/main/software > Acesso em: 15 de Dezembro de 2014. 
BARBOSA FILHO, J.A.D.; VIEIRA, F.M.C.; SILVA, I.J.O. Transporte de frangos: caracterização do microclima na carga durante o inverno. Revista Brasileira de Zootecnia, Viçosa, MG v. 38, n. 12, p. 2442-2446, 2009. Disponível em: $<$ http://www.scielo.br/scielo.php?script=sci_arttext\&pid=S1516-

35982009001200021> Acesso em: 24/05/2016. doi: 10.1590/S151635982009001200021

BARBUT, S. Review: Automation and meat quality-global challenges. Meat Science, v. 96, n.1, p. 335-345, 2014. Disponível em: <http://www.sciencedirect.com/science/article/pii/S0309174013003100> Acesso em: 24/05/2016. doi:10.1016/j.meatsci.2013.07.002

BORGES, S. A.; MAIORKA, A.; Silva, A. V. F. Fisiologia do estresse calórico e a utilização de eletrólitos em frangos de corte. Ciência Rural, Santa Maria, v. 33, n.5, p. 975-981, 2003. Disponível em: <http://www.scielo.br/scielo.php?script=sci_arttext\&pid=S0103-

84782003000500028> Acesso em: 24/05/2016. doi: 10.1590/S010384782003000500028

CANDELAS, F.A., GARCíA, G.J. ; PUENTE, S.; POMARES, J.; JARA, C.A.; PÉREZ, J.; MIRA, D.; TORRES, F. Experiences on using Arduino for laboratory experiments of Automatic Control and Robotics. IFAC-PapersOnLine, v. 48, Issue 29, p. 105110, 2015.2 Disponível em: <http://www.sciencedirect.com/science/article/pii/S2405896315024799> Acesso em: 24/05/2016. doi:10.1016/j.ifacol.2015.11.221

CARVALHO, L. R. M.; AMORIM, H. S. Observando as marés atmosféricas: uma aplicação da placa Arduino com sensores de pressão barométrica e temperatura. Revista Brasileira de Ensino de Física, v. 36, n. 3, p. 1-7, 2014. ISSN 1806-1117. Disponivel em: <http://www.scielo.br/scielo.php?script=sci_arttext\&pid=S1806$11172014000300013 \&$ Ing=en\&nrm=iso> Acesso em: 24/05/2016. doi:10.1590/S1806-11172014000300013

CNA. Confederação da Agricultura e Pecuária do Brasil. Agronegócio: Balanço 2013 - Perspectiva 2014. Instituto CNA, 2013. Disponível em: <http://www.canaldoprodutor.com.br/sites/default/files/001Balan\%C3\%A702013_Per spectivas2014_Web.pdf> Acesso em: 15/02/2014.

FATEHNIA, M.; PARAN, S.; KISH, S.; TAWFIQ, K. Automating double ring infiltrometer with an Arduino microcontroller. Geoderma, v. 262, p. 133-139, 2016. Disponível em: <http://www.sciencedirect.com/science/article/pii/S0016706115300513> Acesso em: 24/05/2016. doi:10.1016/j.geoderma.2015.08.022

HARIKRISHNAN, R. An Integrated Xbee Arduino and Differential Evolution Approach for Localization in Wireless Sensor Networks. Procedia Computer Science, v. 48, p. 447-453, $2015 . \quad$ Disponível em: <http://www.sciencedirect.com/science/article/pii/S1877050915006274> Acesso em: 24/05/2016. doi:10.1016/j.procs.2015.04.118 
IBGE. Instituto Brasileiro de Geografia e Estatística. Produto Interno Bruto Agropecuário do ano de 2012. Disponível em: $<$ http://www.ibge.gov.br/home/estatistica/pesquisas/pesquisa_resultados.php?id_pes quisa=46> Acesso em: 11 de Agosto de 2014.

KAMOGAWA, M. Y.; MIRANDA, J. C. Uso de hardware de código fonte aberto "Arduino" para acionamento de dispositivo solenoide em sistemas de análises em fluxo. Química Nova, São Paulo, v. 36, n. 8, 2013. Disponível em: $<$ http://www.scielo.br/scielo.php?script=sci_arttext\&pid=S0100-

40422013000800023\&Ing=en\&nrm=iso> Acesso em: 24/05/2016. doi: $10.1590 / \mathrm{S} 0100-40422013000800023$

MESAS-CARRASCOSA, F.J.; SANTANO, J D. V.; MEROÑO, E.; DE LA ORDEN, M. S.; GARCÍA-FERRER, A.. Open source hardware to monitor environmental parameters in precision agriculture. Biosystems Engineering, v. 137, n.1, p. 73-83, 2015.

em: <http://www.sciencedirect.com/science/article/pii/S1537511015001208> Acesso em: 24/05/2016. doi:10.1016/j.biosystemseng.2015.07.005

MOLLO NETO, M.; GABRIEL, C. P. C.; SANTOS, V. J. ; ZANETTI, W. A. L. Avaliação de sensores eletrônicos para uso em instrumentos agrometeorológicos alternativos em galpões avícolas. ENCICLOPÉDIA BIOSFERA, Centro Científico Conhecer - Goiânia, v. 11 n. 21; p. 2527, 2015. Disponível em: $<$ http://www.conhecer.org.br/enciclop/2015b/engenharias/avaliacoes\%20de\%20sens ores\%20eletronicos.pdf> Acesso em: 24/05/2016. doi:N/D

MRIDULA, M., SHUKLA, S.R.N. Current wireless sensor nodes (Motes): performance metrics and constraints. International journal of electronic communications engineering, v. 2, n. 1, p. 45-48, 2013. Disponível em: <http://ijarece.org/wp-content/uploads/2013/08/IJARECE-VOL-2-ISSUE-1-4548.pdf>. Acesso em: 24/05/2016. doi: N/D

NÄÄS, I. A.; SEVEGNANI, K. B.; MARCHETO, F. G.; ESPELHO, J. C. C.; MENEGASSI, V.; SILVA, I. J. O. Avaliação térmica de telhas de composição de celulose e betumem, pintadas de branco, em modelos de aviários com escala reduzida. Engenharia Agrícola, Jaboticabal, v. 21, n. 2, p. 121-126, 2001. Disponível em:<http://www.nupea.esalq.usp.br/imgs/publicacoes/44.pdf>. Acesso em: 24/05/2016. doi: N/D

NASCIMENTO, G. R.; PEREIRA, D. F.; NÄAS, I. A.; RODRIGUES, L. H. A.. Índice fuzzy de conforto térmico para frangos de corte. Engenharia Agrícola, n.31, v2, p.219-229, 2011.

Disponível em:<http://www.scielo.br/scielo.php?script=sci_arttext\&pid=S010069162011000200002\&lng=pt\&nrm=iso $>$. Acesso em: doi.org/10.1590/S0100-69162011000200002.

NIENABER, J. A.; HAHN, G. L. Livestock production system management responses to thermal challenges. International Journal of Biometeorology. v. 52, 
p. 149-157, $2007 . \quad$ Disponível em:<http://link.springer.com/article/10.1007\%2Fs00484-007-0103-x\#page-1>. Acesso em: 24/05/2016. doi:10.1007/s00484-007-0103-x

PEREIRA, D.F.; OLIVEIRA, S.C.; PENHA, N.L.J. Logistic regression to estimate the welf are of broiler breeders in relation to environment al and behavioral variables. Engenharia Agrícola, Jaboticabal, v. 31, n. 1, p. 33-40, 2011. Disponível em:<http://www.scielo.br/scielo.php?script=sci_arttext\&pid=S0100-

69162011000100004>. Acesso em: 24/05/2016. doi: 10.1590/S010069162011000100004

SÁNCHEZ, V.; GIL, S.; FLORES, J. M.; QUILES, F. J.; ORTIZ, M. A.; LUNA, J. J.. Implementation of an electronic system to monitor the thermoregulatory capacity of honeybee colonies in hives with open-screened bottom boards. Computers and Electronics in Agriculture, v. 119, p. 209-216, 2015. Disponível em: <http://www.sciencedirect.com/science/article/pii/S0168169915003312> Acesso em: 24/05/2016. doi:10.1016/j.compag.2015.10.018

THOM, E.C. The discomfort index. Weatherwise, Boston, v.12, n.1, p.57-60, 1959. Disponível

em:<http://www.tandfonline.com/doi/abs/10.1080/00431672.1959.9926960>. Acesso em: 24/05/2016. doi: 10.1080/00431672.1959.9926960

VARANIS, M.; LANGONE SILVA, A.; BRUNETTO, P. H. A., GREGOLIN, R. F. Instrumentation for mechanical vibrations analysis in the time domain and frequency domain using the Arduino platform. Revista Brasileira de Ensino de Física, v. 38, n. , p. 1301, 2016. ISSN 1806-1117. Disponível em: <http://www.scielo.br/scielo.php?script=sci_arttext\&pid=S1806-

11172016000100401\&lng=en\&nrm=iso> Acesso em: 24/05/2016. doi:10.1590/S1806-11173812063

WILHELM, L.R. Numerical calculation of psycrometric properties in SI units. Transactions of the ASAE, 1976. Disponivel em:<https://elibrary.asabe.org/abstract.asp?aid=36019\&t=2\&redir=\&redirType=> Acesso em: 24/05/2016. doi: 10.13031/2013.36019 